\title{
Recommended changes to Interval Management to Achieve Operational Implementation
}

\author{
Brian Baxley, Kurt Swieringa, Roy Roper \\ NASA Langley Research Center \\ Hampton, VA 23681
}

\author{
Clay Hubbs, Paul Goess, Richard Shay \\ National Institute of Aviation \\ Hampton, VA 23666
}

\begin{abstract}
A 19-day flight test of an Interval Management (IM) avionics prototype was conducted in Washington State using three aircraft to precisely achieve and maintain a spacing interval behind the preceding aircraft. NASA contracted with Boeing, Honeywell, and United Airlines to build this prototype, and then worked closely with them, the FAA, and other industry partners to test this prototype in flight. Four different IM operation types were investigated during this test in the en route, arrival, and final approach phases of flight. Many of the IM operations met or exceeded the design goals established prior to the test. However, there were issues discovered throughout the flight test, including the rate and magnitude of IM commanded speed changes and the difference between expected and actual aircraft deceleration rates.
\end{abstract}

Keywords - interval management, air traffic management, flight test, avionics.

\section{INTRODUCTION}

The Air Traffic Management Technology Demonstration (ATD-1) project conducted a 19-day flight test in early 2017 with the goals of developing an Interval Management (IM) avionics prototype, and integrating the prototype into two test aircraft to conduct validation flights. Boeing Research and Technology, Boeing Commercial Aircraft, Honeywell, and United Airlines, developed comprehensive plans for the flight test $[1,2]$. Three aircraft were used to fly IM operations in highaltitude en route airspace, IM arrival operations from en route altitudes to the final approach fix, and IM operations intercepting final approach within the terminal airspace.

Preliminary results indicate the IM avionics prototype and procedures enabled pilots to achieve the desired spacing interval for the majority of the operations $[3,4]$. However, many operations contained speed control behaviors or required actions by the flight crew that would likely be unacceptable to line pilots during typical day-to-day operations. Examples include the high rate of speed commands and speed reversals (speed increases followed by speed decreases), pilot workload associated with managing speed and altitude when using speed intervene mode, speed increases prior to the end of the IM operation, and the aircraft's actual deceleration not aligning well to the IM avionics prototype's expected deceleration rate.

This paper describes the ATD-1 concept, relevant results from previous IM research, the design of the flight test, flight test results, case studies root cause of behaviors requiring improvement, and recommendations for future development.

\section{ATD-1 CONCEPT OF OPERATIONS AND PROCEDURES}

The ATD-1 concept of operations combines advanced arrival scheduling, controller decision support tools, and aircraft avionics to improve the efficiency of arrival operations into busy airports $[5,6]$. Speed control, instead of vectoring, is used to achieve either the desired schedule or spacing intervals between aircraft. The IM avionics and procedures support the ATD-1 concept by enabling en route controllers to issue a single strategic clearance to the flight crew to achieve a specific time or distance behind the preceding aircraft (the Target aircraft) by the Achieve By Point (ABP), and then maintain that interval until the Planned Termination Point (PTP). The IMequipped aircraft conducting the IM operation (Ownship) has onboard avionics that uses the aircraft's flight data (destination, route of flight, current location, forecast and sensed winds) and the Target aircraft's state data (position, altitude, heading) transmitted via Automatic Dependent Surveillance-Broadcast (ADS-B) to calculate the airspeed necessary for the Ownship to achieve the desired spacing interval.

Based on the air traffic management goal, the ATD-1 concept has the controller issue a clearance for one of four different IM operations depending on the air traffic goal:

- "Maintain" has the Ownship maintain the current spacing interval behind the Target aircraft until the PTP. The onboard IM avionics calculates the Assigned Spacing Goal (ASG) based on the current interval.

- "Capture" has the Ownship translate fore or aft of the current spacing interval at or faster than a minimum closure rate to the controller assigned ASG, then maintains that spacing interval to the PTP.

- "Cross" has the Ownship translate fore or aft so the ASG is met by the ABP and then maintained until the PTP. This operation can occur with aircraft on merging routes, while Maintain and Capture require the aircraft to be in-trail on the same route.

- "Final Approach Spacing" is a subset of the Cross operation, but is initiated in terminal airspace. This operation can begin when one aircraft is established on final approach and the other aircraft is either established on final approach, or on an intercept track to the final approach course. 


\section{RELEVANT RESULTS FROM PREVIOUS IM RESEARCH}

This section briefly describes previous IM research relevant to the accuracy of IM operations, rates of IM commanded speed changes, and pilot procedures used to conduct the IM operation.

\section{A. Interval Management with Spacing to Parallel Dependent Runways (IMSPiDR)}

The IMSPiDR simulation [7] explored IM operations to dependent parallel runways at the Dallas-Fort Worth airport. Requirements unique to simultaneous dependent approach operations were of particular interest [8, para. 5-9-6]. An earlier version of the speed control law was used in the experiment, and controller pilot data link communication (CPDLC) was used to communicate the IM clearance.

Three groups of eight pilots flew both desktop and full-scale simulators in ten scenarios. Five of the scenarios investigated the use of speed control to achieve a required time of arrival (RTA) at the runway. The remaining five scenarios investigated the use of the RTA algorithm enhanced with IM to achieve the requirements for dependent parallel operations. This resulted in 88 RTA operations, and 89 RTA plus IM operations.

Results and conclusions identified in IMSPiDR included:

- The mean spacing accuracy at the runway threshold for operations that only used the RTA was 3.5 seconds ( $S D=4.0$ seconds), and the mean spacing accuracy for operations with RTA enhanced with IM was 2.2 seconds or less $(S D=3.9$ seconds).

- $\quad$ The 25-minute IM arrival operations required 5 to 11 more speed changes than non-IM operations flown by the same flight crew on the same routes.

- $\quad$ Pilots rated the workload of conducting IM operations using CPDLC as easy (1.97 on the Modified CooperHarper (MCH) scale of 1-very easy to 10-impossible).

- The crews expressed a desire for fewer and more predictable IM speed changes.

- $\quad$ The results suggested the published arrival procedures should be designed with slightly shallower descent angles and slower speeds to enable the use of speed as the primary control mechanism.

\section{B. Research and Procedureal Testing of Routes (RAPTOR)}

The RAPTOR experiment [9] explored IM operations to a single runway at Phoenix Sky Harbor International Airport. Since the avionics were designed to support a near-term retrofit implementation, voice instructions from controllers instead of CPDLC was used to transmit the IM clearances. Eight pilots participated in the experiment, and used the same simulators as in IMSPiDR. Due to schedule constraints and inclement weather, only 16 operations were completed. Results and conclusions identified in RAPTOR included:

- The mean spacing precision at the final approach fix was 2.3 seconds ( $S D=5.8$ seconds).

- $\quad$ Flight crews rated the workload of IM operations when using voice instructions as acceptable (1.9 on the $\mathrm{MCH}$ workload rating scale of 1 -very easy to 10 -impossible).
- Flight crews rated IM operations when using voice instructions as acceptable (5.9 on a scale of 1completely unacceptable to 7-completely acceptable).

- Flight crew responses indicated the high frequency of IM speed command changes and speed reversals compared to current operations were problematic.

- The behavior of the IM software is highly coupled to the behavior of the Target aircraft. Cases where the Target aircraft decelerates at locations other than the published deceleration point can trigger additional IM commands.

\section{Interval Management with Alternative Clearances (IMAC)}

The IMAC experiment [10] examined IM operations to a single runway at the Denver International Airport. Similar to RAPTOR, controllers used voice communications to provide IM clearances. Twenty-four pilots flew 79 IM north- or south-flow arrival operations using the same simulators as RAPTOR. Results and conclusions identified in IMAC included:

- The mean spacing precision at the final approach fix (FAF) was 1.0 seconds ( $S D=6.3$ seconds).

- Flight crews rated the workload to conduct IM operations slightly higher than non-IM operations $(2.5$ vs. 1.4 on a $\mathrm{MCH}$ workload rating scale ranging from 1very easy to 10 -impossible).

- $\quad$ Flight crews rated IM operations as acceptable (5.6 on a scale of 1-completely unacceptable to 7-completely acceptable).

- $\quad$ Flight crew responses indicated the high frequency of IM speed command changes and speed reversals were problematic.

- $\quad$ Some flight crews reported using the vertical navigation speed mode to conduct IM operations was challenging, and if the IM system commanded a speed decrease just prior to an altitude constrained waypoint, there was potential to miss the altitude constraint.

\section{FLight TEST DESIGN}

\section{A. Aircraft and Pilots Participating in the Flight Test}

The ATD-1 flight test took place in Seattle Air Route Traffic Control Center (ARTCC) and Moses Lake Terminal Radar Approach Control Facility (TRACON) airspace. The participating aircraft were a Falcon 900 (F900) and a Boeing 757 (B757) provided by Honeywell, and a Boeing 737 (B737) provided by United Airlines. The B757 and B737 were equipped with the IM avionics prototype, while the non-IM equipped F900 was used as a Target aircraft only. All three aircraft were equipped with ADS-B Out and Global Navigation Satellite System (GNSS).

The pilots for all three aircraft were provided by their respective flight departments, and were current and qualified for the duties they performed. All of the pilots of the two IM equipped aircraft and the two Boeing flight test directors completed a NASA created computer-based training program, and then attended academic classes and simulator training at the NASA Langley Research Center prior to the flight test [11]. 


\section{B. Air Traffic Control Facilities}

Five air traffic control facilities were involved in the daily operation of the ATD-1 flight test. The tower facilities at Boeing Field (KBFI) and Seattle-Tacoma International Airport (KSEA) coordinated with the Seattle TRACON facility to synchronize the departure times of the three aircraft to setup the first highaltitude en route operation. Prior to reaching en route altitudes, the Seattle TRACON controllers handed off the flight crew to the Seattle ARTCC controllers.

The en route IM operation and the first half of each arrival IM operation was conducted within Seattle ARTCC airspace. The second half of the arrival IM operation, the climb out after the missed approach, and the final approach spacing operations was conducted within Moses Lake TRACON airspace.

Upon completion of the test conditions for the day, Moses Lake TRACON facilitated the return of the three aircraft to their respective airports with Seattle ARTCC and Seattle TRACON.

\section{Design of ATD-1 Arrival and Approach Procedures}

Custom built Standard Terminal Arrival Routes (STARs) connected to Required Navigation Performance Authorization Required (RNP AR) procedures used in this flight test, allowing the aircraft to be flown using Performance Based Navigation procedures throughout the entire IM operation. Of note was the RNP AR approach contained a radius-to-fix (RF) turn, which is a relatively new procedure in today's airspace, and not normally flown by some of the pilots involved in the flight test. These procedures were intended to be representative of other such procedures, and testing of these procedures in different simulators prior to flight provided the expected performance.

However, during the flight test it became apparent that when using speed as the primary control mechanism to achieve the spacing interval, the speeds and altitudes of the custom STARs required more than normal use of spoilers. Additionally, some waypoint speed constraints were slower than desired causing aircraft to fly at slower than desired speeds below 18,000 feet. This can be seen in the top panel of Fig. 1 for the SUBDY1 STAR where the nominal or published speed (solid black line) of 270 knots at $40 \mathrm{nmi}$ is faster than normal, while the 210 knots at $30 \mathrm{nmi}$ is slower than normal. The IM algorithm was allowed to deviate up to $\pm 15 \%$ from that speed to achieve the desired spacing interval (dotted black lines in Fig. 1).

Conversely, the implementation of expected IM speeds during the RNP AR approach caused some of the aircraft to be fast on final. This was especially prevalent on the UPBOB STAR shown in the bottom panel of Fig. 1, where the nominal speed at $10 \mathrm{nmi}$ from the final approach fix is 240 knots. Furthermore, the use of RF turns in the RNP AR approach is relatively new to the IM research community, and their interaction with straight-in approaches has not been studied. As a consequence, some of the speed constraints established during the planning phase of the flight test negatively influenced the IM commanded speed rate of change and the pilot responses.

In addition to sub-optimum speed constraints at some waypoints, the magnitude of the procedural changes during the flight test created additional challenges impacting the spacing accuracy of the IM prototype (described in the Results section).

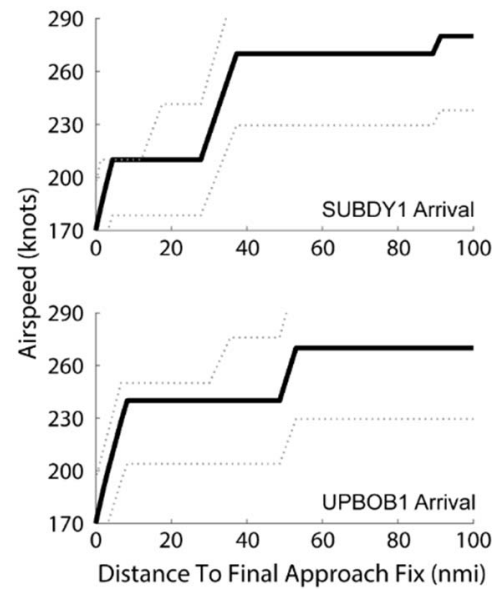

Fig. 1. Nominal speeds of the SUBDY1 and UPBOB1 STARs.

\section{Scenario Description}

The test matrix consisted of thirty-eight scenarios designed to evaluate the IM avionics performance in three different phases of flight. Each scenario specified the route of flight for the Target and Ownship, type of IM operation to be performed, and the initial spacing error to be resolved. The scenario routes shown in Fig. 2 are high altitude en route operations (blue), arrival operations from en route altitude to the decision altitude of the instrument approach (red), and final approach operations initiated within the airport's terminal airspace (purple).

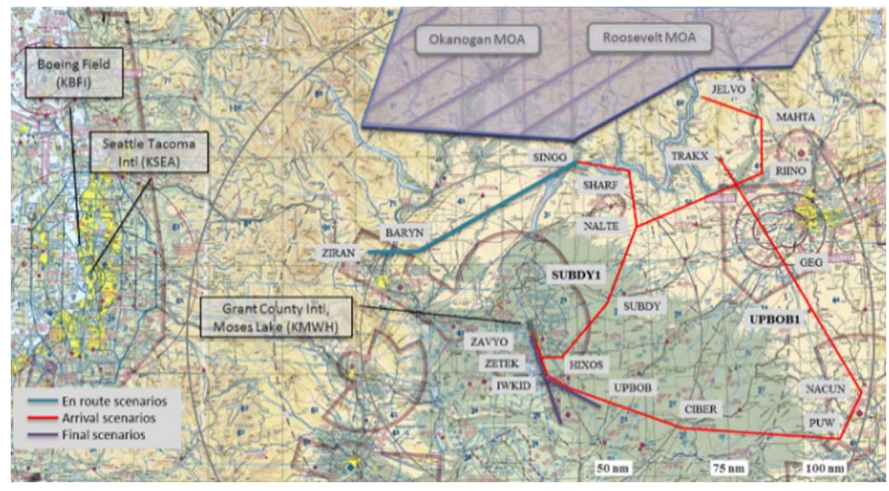

Fig. 2. Waypoints and routes used during flight test.

Maintain and Capture operations were conducted when both aircraft were in-trail on the en route portion of the route, or intrail on either of the SUBDY1 arrivals. Cross-Merge operations were conducted when the Target and Ownship were on opposite transitions of the SUBDY1 route, with NALTE set as the ABP. Cross-FAF operations were conducted when the Target was on either SUBDY1 route and the Ownship was on the UPBOB1 route, or conducted with the Target on one SUBDY1 route and the IM-equipped aircraft on the other SUBDY1 route, with ZAVYO (the FAF) set as the ABP. Final approach spacing operations consisted of one aircraft established on final (either the Target or the Ownship) and the other aircraft either established on final or on an intercept to final. SINGG was used as the PTP for en route IM operations, and ZAVYO (the FAF) was used as the PTP for all other IM operations. 
During the morning briefing, flight crews and controllers received the daily sequence of selected scenarios, and were given test cards containing call sign, route of flight, desired initial airspeeds, and IM-specific information. Since the two ground components of the ATD-1 concept were unavailable for this flight test, the IM clearance information was precoordinated and published on the test card instead of verbally issued by the air traffic controllers. In particular, the ASG was generated by the flight crew and flight test director using the spacing interval calculated by the IM prototype just prior to initiation of the operation (the cyan "167" in Fig. 3), plus the spacing error defined in the test matrix for that scenario.

\section{E. Prototype IM Equipment}

Fig. 3 shows the electronic flight bag (EFB) which hosted the IM application during the flight test [12]. Each pilot had their own EFB, mounted on the outboard panel of the cockpit. Each EFB had a touchscreen for data entry, displayed the entered data and data from the IM avionics, displayed surrounding air traffic for awareness, and provided information to the configurable graphics display (CGD).

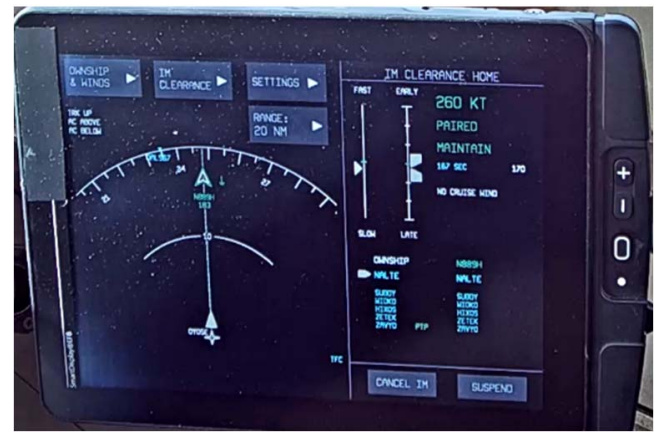

Fig. 3. Electronic flight bag.

The CGD was mounted within each pilot's primary forward field of view, either above the glareshield (Fig. 4) or next to the primary flight display [13]. The CGD repeated essential information needed to conduct the operation, in particular the IM commanded speed, fast/slow indicator, and IM messages.

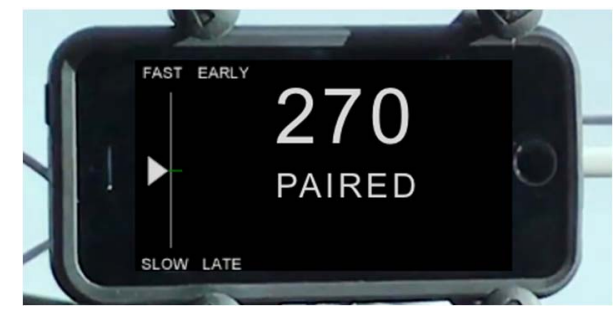

Fig. 4. Configurable graphics display.

\section{F. IM Speed Control Laws}

A trajectory-based operation (TBO) speed control law and a state-based constant time delay (CTD) speed control law were implemented in the IM prototype [14-17]. The TBO speed control law is used for the achieve-stage of the Cross operation and the final approach spacing operation. The CTD speed control law is used for the maintain phase of the Cross operation, the Capture operation, and the Maintain operation. In general, both control laws calculate a spacing error, a speed correction based on the spacing error, and the speed command as the sum of the nominal speed at that point of the published procedure and the speed correction.

The TBO speed control law calculates the spacing error as the difference between the ASG and the predicted spacing interval, which is calculated using trajectory information from the Ownship and Target aircraft. The IM commanded speed is the sum of the Ownship's nominal trajectory speed, a proportional control term to null the spacing error, and a ground speed compensation term used to prevent high closure rates between the Target and Ownship. For a time-based ASG, the spacing error is calculated differently before and after the Target aircraft crosses the ABP. Before the Target aircraft crosses the $\mathrm{ABP}$, the spacing error is calculated using the timeto-go of the IM aircraft along its trajectory $\left(T T G_{I M}\right)$, the timeto-go of the Target aircraft along its trajectory $\left(T T G_{T G T}\right)$, and the $\operatorname{ASG}(\Delta)$.

$$
e(t)=T T G_{I M}(t)-T T G_{T G T}(t)-\Delta
$$

When the Target aircraft crosses the ABP, its crossing time is recorded and the objective of the IM aircraft is to arrive at the ABP $\Delta$ seconds after the Target aircraft. To accomplish this goal, the spacing error is defined using the time-to-go of the Ownship ( $\left.T T G_{I M}\right)$, the time when the Target aircraft crossed the $\operatorname{ABP}\left(t_{T G T_{A B P}}\right)$, the current time $(t)$, and the ASG $(\Delta)$.

$$
e(t)=T T G_{I M}(t)-\left(t-t_{T G T} T_{A B P}-\Delta\right)
$$

The CTD speed control law calculates the spacing error as the difference between the ASG and the measured spacing interval. For time-based spacing goals, calculating the measured spacing interval relies on historical state data from the Target aircraft, and requires the Ownship to be on the same route as the Target aircraft. The measured spacing interval is the time elapsed since the Target aircraft crossed the Ownship's current along-path position, and the spacing error is the difference between the measured spacing interval and the ASG.

$$
e(t)=t-t_{T G T}\left(x_{I M}(t)\right)-\Delta
$$

Here, $x_{I M}(t)$ is the along path Ownship position at time $t$, and $t_{T G T}\left(x_{I M}(t)\right)$ is the time when the Target was at the Ownship's current position. Proportional control is used to determine the amount of speed required, which is added to the Target aircraft's ground speed that was flown at the Ownship's current position, converted to an airspeed.

The IM commanded speed produced by the control laws is limited before being displayed to the pilots. Limits to the commanded speed included the maximum operating speed, a $\pm 15 \%$ boundary around the Ownship's trajectory speed (based on the published procedure), and regulatory speed restrictions. To reduce the number of IM speed changes, hysteresis is applied to the commanded speed, and the speed is discretized to a value of 5 knots when the Ownship is close to the PTP and 10 knots when the Ownship is not close to the PTP. 


\section{G. Flight Crew Procedures for an IM Operation}

The flight crew learned and practiced IM data entry and IM operation procedures during the simulator training at NASA Langley [11], and used those procedures during the flight test.

In the data entry phase of the IM operation, the flight crew used the EFB to enter information about the Ownship's route and destination, forecast en route and descent winds, and the IM clearance itself. The Ownship and wind information could be entered anytime, while the IM clearance information was entered at the beginning of each scenario.

In the operational phase of IM, the flight crew procedure was to set the IM commanded speed from the IM prototype (Fig. 3 and 4) into the airspeed window of the mode control panel. This procedure was designed to be similar to entering an airspeed issued via voice instruction from the controller. Given the capabilities of the flight management computers of the two IMequipped aircraft, this meant the aircraft was in the vertical navigation (VNAV) speed mode, and controlled pitch to achieve the new IM commanded speed (increase the descent rate to accelerate or decease the descent rate to decelerate).

This flight management mode for controlling speed, termed speed intervene mode, is typically avoided by commercial airline pilots when conducting arrival operations similar to those used in this flight test. This is due to the reduced altitude protection this speed mode provides (only protects for AT OR ABOVE altitude restrictions), and the increased use of the throttles and spoilers to maintain the required path.

\section{Flight Test Results}

This section describes the performance of the IM avionics prototype, the pilots' assessment of the acceptability of conducting IM operations, and the characteristics of the speed behavior observed throughout the flight test.

\section{A. Spacing Accuracy of IM Avionics Prototype}

An important performance metric is how close the IM aircraft was to the ASG at the end of the IM operation, which was the last waypoint (SINGG) for the en route operations, and the final approach fix (ZAVYO) for the arrival and final approach spacing operations. For time-based operations, this is defined as the difference between the ASG and the difference in time between when the Target aircraft and Ownship crossed the PTP. Operationally, the maintain stage goal is a spacing error at the PTP within 10 seconds, $95 \%$ of the time. This corresponds to a $S D=5$ seconds if the data are normally distributed.

Fig. 5 shows that the time-based arrival scenarios surpassed the performance criteria at the PTP for the Maintain, Capture, and Cross-Merge operations. The mean spacing error for all maintain operations at the PTP was within 2 seconds and the standard deviation was less than 3 seconds. The Maintain operations had an average of -1.13 seconds $(S D=2.99, N=18)$, Capture operations an average of 0.55 seconds $(S D=2.63$, $N=32$ ), and Cross-Merge operations an average of -0.47 seconds $(S D=2.45, N=27)$ [18]. For clarity, the negative average indicates the spacing interval was less than the ASG; that is, the aircraft was early or ahead of the desired location.

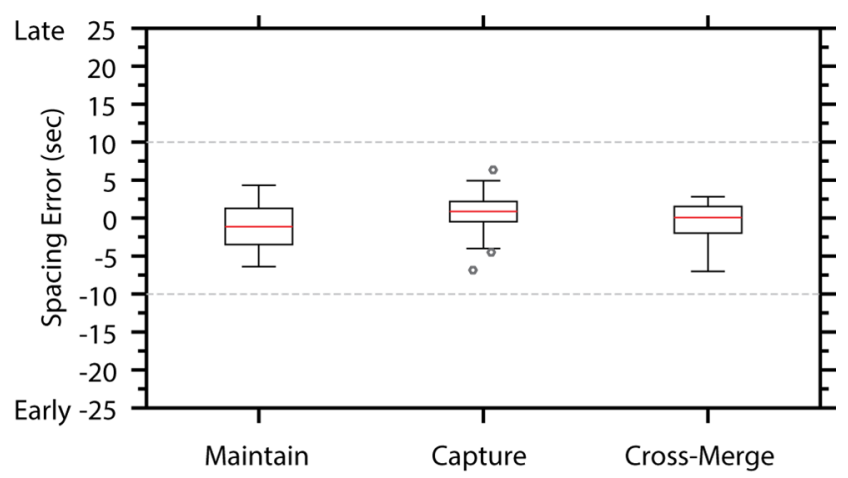

Fig. 5. Accuracy of time-based maintain stage arrivals.

These results indicate the ability of the maintain stage of these IM operations to precisely meet the ASG at the PTP. These results are consistent with a field evaluation conducted by UPS, MITRE, and the FAA in 2010 that found aircraft that followed the IM commanded speeds were able to obtain spacing accuracies within 8 seconds [17].

A more comprehensive description of the IM prototype performance during the flight test is given in [3], including distance-based spacing operations, en route operations, final approach spacing operations, and those operations that included an achieve phase.

Of interest in [3] was the spacing performance at the ABP for Cross operations where the ABP and PTP were co-located at the FAF. The spacing accuracy of these operations was noticeably worse than the spacing performance at the PTP for operations that included a Maintain stage (i.e., the operations described in Fig. 5). Factors that contributed to the poor spacing accuracy were procedure design, differences between the expected versus actual deceleration rate, and a software issue where the sensed wind was not used to update the estimated time of arrival as intended.

While the spacing performance of IM operations in the Achieve phase (TBO control law) were not as good as the IM operations in the Maintain phase (CTD control law), the IM commanded speed rate when using the TBO control law was much lower when compared to the CTD control law (described in greater detail in sub-section $\mathrm{C}$ of this part).

\section{B. Pilot Responses to Acceptability of IM Operation}

During IM operations, pilots are provided IM commanded speeds to achieve the assigned spacing interval. During these operations, the commanded speeds should generally be logical to the pilots, otherwise the pilots may lose trust in the system and elect to not conduct the IM operation. Therefore, at the end of each scenario the pilots rated the operational acceptability of the IM speed using a Likert scale that ranged from ' 1 ', (completely unacceptable) to ' 7 ' (completely acceptable). The goal was a mean rating greater than or equal to ' 5 '.

Results of the statistical analysis shown in Table 1 indicate that flight crews found the IM speeds to be overall operationally acceptable, with the mean operational acceptability ratings of the IM speeds statistically significantly greater than ' 5 ' for all clearance types $(\mathrm{p} \leq 0.025)$. 
TABLE I. ACCEPTABILITY OF IM SPEED

\begin{tabular}{|c|c|c|cc|}
\hline \multirow{2}{*}{ Scenario } & Clearance & \multirow{2}{*}{$N$} & \multicolumn{2}{|c|}{ IM Speed } \\
& Type & Mean & $S D$ \\
\hline \multirow{3}{*}{ En route } & Maintain & 13 & 6.7 & 0.5 \\
& Capture & 6 & 6.5 & 0.5 \\
\hline \multirow{3}{*}{ Arrival } & Maintain & 31 & 5.5 & 1.3 \\
& Capture & 58 & 5.9 & 0.9 \\
& Cross & 137 & 5.6 & 1.2 \\
\hline Final & Final & 15 & 6.5 & 0.5 \\
\hline
\end{tabular}

Although the flight crews rated the IM speeds as operationally acceptable, several important issues were identified in the comments associated to those ratings. The comments frequently indicated that the IM speeds were acceptable for the flight test but not for normal daily flight operations. The issues reported by the flight crews can be separated into categories of speed command behavior, procedure design, deceleration rates, and the flight management system mode used when conducting IM operations.

Throughout the flight test, the pilots reported several instances where the IM commanded speed behavior was undesirable. The particular behaviors found undesirable were multiple speed changes within a short period of time and speed reversals. Both the speed change rate and number of speed reversals are described in more detail in subsequent sections.

The speed constraints on the arrivals and approaches developed for this flight test had characteristics that pilots found undesirable. These speed constraints created large deceleration segments, such as the 270 to 210 knot deceleration on the SUBDY 1 arrival, and the 240 to 170 knot deceleration on the UPBOB1 arrival. Additionally, the speed constraints on the approach procedures resulted in nominal IM commanded speeds that were faster than desired when close to the FAF. In the cases where the spacing interval was smaller than the ASG, the IM commanded speeds where even faster than desired by pilots.

The third category of responses stated the deceleration rate used by the IM prototype did not appear to be accurate at some altitudes and aircraft configurations, which in turn generated additional speed commands to compensate for that difference. This mismatch between deceleration rates was amplified by the magnitude of the procedural speed changes (i.e., the 240 to 170 knot deceleration on the UPBOB1 arrival). A similar behavior (mismatch) occurred during accelerations, primarily at en route altitudes, where the aircraft's actual acceleration was less than expected by the IM avionics.

Finally, when conducting IM operations, the pilot procedure was to manually enter the IM commanded speeds into the aircraft's mode control panel speed window. For the IM equipped aircraft used in the flight test, this causes the aircraft to use pitch control to maintain speed instead of the vertical path. Pilots are therefore required to manually manipulate the throttles and spoilers to maintain the desired vertical path, which the pilots reported that the level of intervention required while flying the IM operation was frequently too high. Additionally, a characteristic unique to the speed intervene mode is the aircraft is no longer protected from flying higher than an AT OR BELOW altitude constraint. This is problematic for normal daily operations since the pilots must now manually manipulate throttles and spoilers to achieve the correct vertical velocity to remain on a vertical path to meet that constraint.

\section{IM Commanded Speed Change Rate}

The IM commanded speed change rate is the number of speed commands per minute that the IM avionics presented to the pilots, who then manually entered them into the mode control panel speed window. This metric is used as an indirect measure of pilot workload. For current day arrival operations without metering in effect, the rate of speed changes is driven by the number of speed constraints on the published procedure, company operating procedures, and controller instructions. Since non-IM operations were not conducted during the ATD-1 flight test, an approximation can be made using Fig. 1 where three speed changes happen during the 20 minutes required to fly the STAR (includes the 250 knot below 10,000 feet restriction not shown in Fig. 1). Based on company operating procedures, there are typically three speed changes during the 10 minutes required to fly the RNP AR approach. This equates to approximately 0.15 changes per minute on arrival and 0.30 changes per minute on approach.

The speed command rate during the flight test (Fig. 6) was significantly higher during IM operations relative to current day operations. By operation type, the mean speed command rate for Maintain operations was 0.80 speed changes per minute $(S D=0.23, N=18)$, Capture operations was $0.54(S D=0.18$, $N=32)$, Cross-Merge operations was $0.64(S D=0.15, N=27)$, and Cross-FAF operations was $0.45(S D=0.15, N=41)[3,18]$.

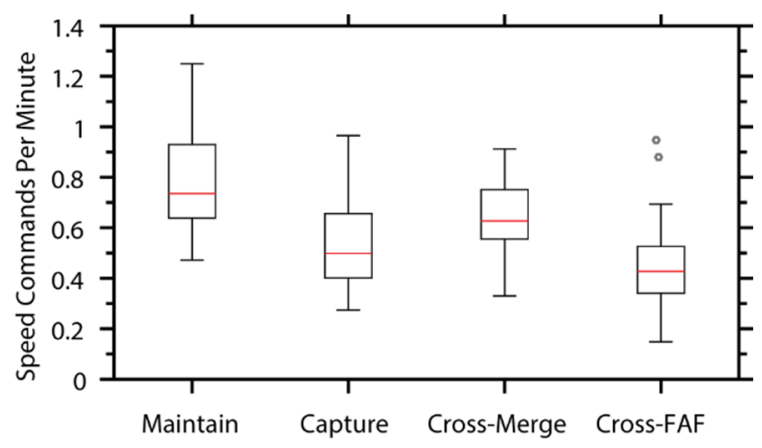

Fig. 6. IM commanded speed change rate by IM type.

The average speed command rate of the Maintain operation, which used the CTD speed control law, had the largest speed command rate and the Cross-FAF operation, which used the TBO speed control law, had the lowest speed command rate [Fig. 6]. The Capture Operation also used the CTD speed control law, but there were heuristics used when the aircraft was capturing the desired spacing goal to minimize the speed command rate. The Cross-Merge operation used the TBO speed control law for the first portion of the arrival and the CTD Speed control law for the second part, therefore its speed change rate was higher than the Cross-FAF operations. The speed commands are analyzed in greater detail in upcoming sections to better understand the time between speed changes, the speed change magnitude, and the number of speed reversals. 


\section{Time Between IM Commanded Speed Changes}

The speed command rate described in the previous section provides useful information about the average speed command rate across the entire IM operation; however, it does not capture cases where there were several speed commands within a short period of time. The time between consecutive speed commands was examined in order to better understand how often the time between speed commands was small (i.e., less than 30 seconds).

Fig. 7 shows an empirical cumulative distribution function for the time between consecutive speed commands. Fig. 8 is an enlarged figure showing the subset of the empirical cumulative distribution function where the time between consecutive speed commands was 60 seconds or less. The results indicate that the Maintain clearance was more likely to have a shorter time between speed commands than the other clearances, whereas the Cross-FAF clearance was more likely to have a longer time between speed commands. Approximately $30 \%$ of the speed commands during Maintain operation occurred within 30 seconds of the previous speed command. In contrast, only $13 \%$ of the speed commands during the Cross-FAF operations occurred within 30 seconds of the previous speed command. One potential explanation for this difference is that Maintain operations use the CTD speed control law, which attempts to match the time history ground speed of the Target Aircraft, whereas the Cross-FAF operation uses the trajectory-based speed control law, which is less susceptible to Target Aircraft ground speed fluxuations.

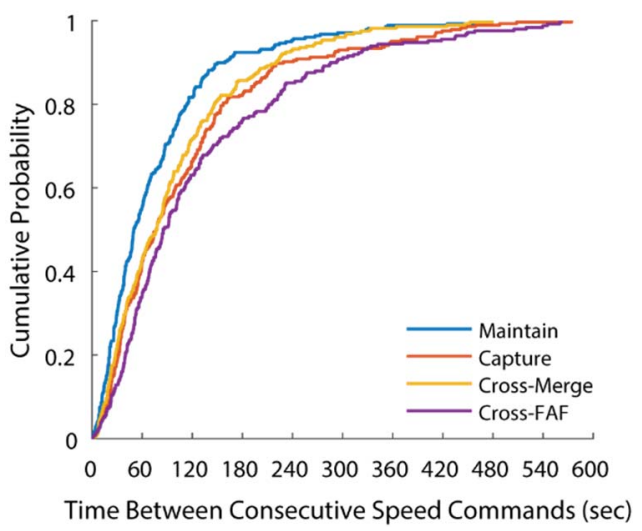

Fig. 7. Cumulative probability of time between consecutive speed commands.

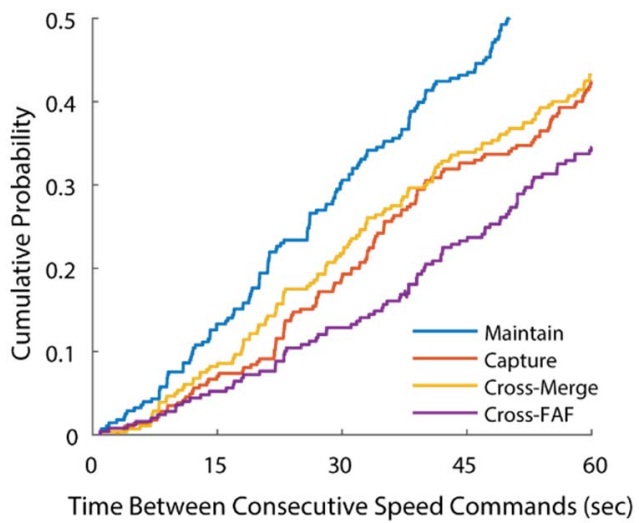

Fig. 8. Cumulative probability of time between consecutive speed commands (enlarged to equal to or less than 60 seconds or 0.5 probability).

\section{E. Magnitude Of IM Commanded Speed Change}

Another metric used to analyze the IM commanded speed behavior throughout the operation is the magnitude of the speed change, measured as the difference between the previous commanded speed and the new commanded speed. Negative values indicate a speed decrease and positive numbers indicate a speed increase.

Fig. 9 show the magnitude of the IM speed changes by IM operation type. A majority of the speed changes had a magnitude between -15 and 15 knots, with the overwhelming majority of those changes either +10 knots or -10 knots since the IM speeds are discretized in 10 knot intervals. Most large speed decreases were associated with the published procedure, such as 270 to 210 knot speed decrease on the SUBDY1 arrival and the 240 to 170 knot speed decrease on the UPBOB1 arrival (see Fig. 1).

Of particular interest is the number of speed increases, shown by the yellow portion of the bars in Fig. 9. During typical arrival operations, it is desirable for the airspeeds flown by an aircraft to be monotonically decreasing. While it may not always be possible to avoid speed reversals to meet the spacing objectives, the IM algorithm should ideally be designed to avoid increasing speed during a descent unless absolutely necessary. The data in Fig. 9 indicates that the Maintain Operation had an approximate average of 7 speed increases per arrival while the Cross-FAF operation had an approximate average of 3 speed increases per arrival.

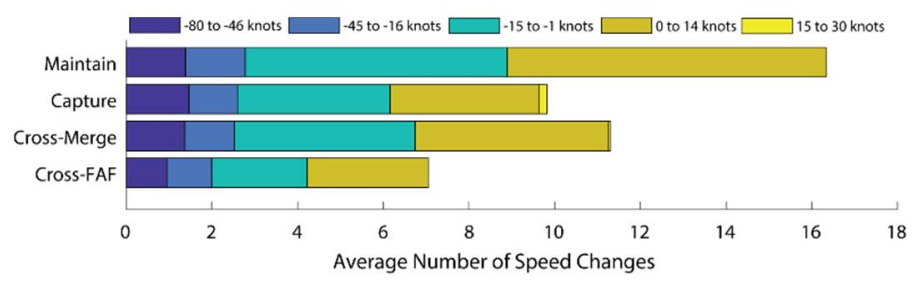

Fig. 9. IM commanded speed change magnitude by IM operation type.

In general, the magnitude of the IM speed change was rated by the pilots as acceptable, except for the large magnitude speed changes of greater than 40 knots on arrival and 20 knots on approach that were due to the design of the procedures.

\section{F. Speed Reversals (Speed Trend Changes)}

Pilot comments indicated that instances where the IM commanded speed reverses (the trend is changed) reduced the acceptability of the IM operation. The number of speed reversals was examined in order to understand how frequently they occurred. For the purposes of this analysis, a speed reversal is defined as a speed increase when the previous speed command was a speed decrease, or a speed decrease when the previous speed command was a speed increase. While some speed reversals may be required to meet the spacing objectives, they should be minimized to the greatest extent possible in order to maximize the efficiency of the IM operation and provide pilots with predictable speeds.

Fig. 10 shows the number of speed reversals observed for all of the time-based arrival operations in the flight test. Between $44 \%$ to $47 \%$ of the speed commands for each of the IM operation types were reversals. The Maintain operation had 
the highest number of speed change reversals in addition to the highest number of speed changes as noted in the previous section. The relatively high percentage of speed changes that were reversals for all IM types supports the pilot feedback that the rate of IM commanded speed reversals was too high.

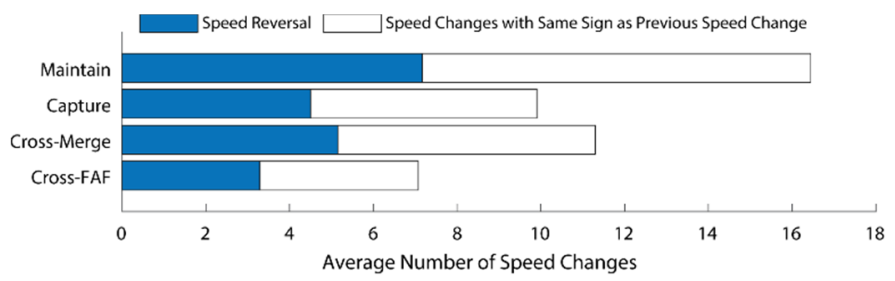

Fig. 10. Percentage of speed reversals by IM operation type.

\section{CASE STUdies}

The previous section described pilot comments indicating that the acceptability of the IM commanded speeds would be substantially improved if behaviors such as frequent speed changes and speed reversals could be reduced. Descriptive statistics were used to understand how often these speed behaviors occurred, and the results supported the comments that these less than desirable behaviors must be reduced prior to implementation in real-world operations. In this section, case studies are presented to explain some of the root causes of these less than desirable speed behaviors.

\section{A. Case Study 1: Speed Increases when Capturing the ASG}

The first behavior is the increase in the IM commanded speed capturing the ASG during a Capture operation (CTD control law). At the initiation of the Capture operation in Fig. 11, the IM equipped aircraft has 35 seconds of spacing error (green line) to correct. Since the CTD speed control law is used, the spacing error is corrected by commanding a slower IM speed (blue line) than the Target aircraft's time history speed (grey line); that is, the ground speed that the Target aircraft was flying when it was at the Ownship's current position, converted to an airspeed.
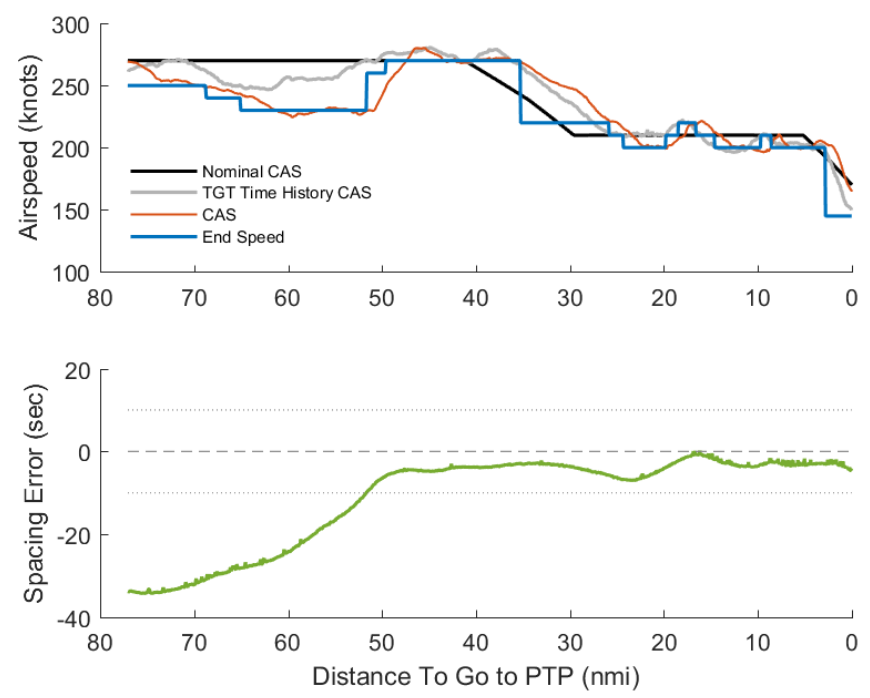

Fig. 11. Increasing IM commanded speed when capturing ASG.
Due to the initial spacing error and changes in the Target aircraft's speed, the commanded speed changes from 250 to 240 then 230 knots (blue line) at 70 to $65 \mathrm{nmi}$ distance to go. Once the spacing error is almost resolved at $52 \mathrm{nmi}$ (green line), the IM commanded speed increases to 260 knots, then again to 270 knots as the Target aircraft speed increases from 250 to 260 knots (grey line).

Since spacing error is corrected by deviating from the Target aircraft's time-history speed, the Capture operation commands a speed decrease to correct an early spacing error. As the error is nulled, the commanded speed will return to the Target aircraft's time-history speed, causing a series of speed increases.

At least two options should be explored to reduce the need for speed increases as the spacing error approaches zero. The first is to use the Capture operation only when the spacing error is relatively small. This would reduce the speed deviations from the Target aircraft's speed that are needed to correct the spacing error. This conclusion aligns with the current concept of operations. A second option would be to use a Cross operation, since it enables the use of predictive TBO speed control laws that could utilize upcoming deceleration segments to assist in reducing speed changes and speed increases.

\section{B. Case Study 2: Frequent Speed Commands}

A second behavior is the high frequency of speed commands that occurs primarily during the Maintain phase of an operation (CTD control law). The Maintain operation shown in Fig. 12 illustrates how any change in the spacing error (green line), or changes in the Target aircraft's time-history speed, causes an immediate IM commanded speed change (blue line). In this case study, the result was 25 speed commands during the 22-minute operation. Causes for these changes to the spacing error can be differences between the headwind component experienced by the Target and Ownship (which can occur when the aircraft are on the same path but at different altitudes), changes to the Target aircraft's airspeed (dependent on precision of that aircraft's auto-throttle system), or changes to the aircrafts' ground track as it continues along the published procedure.
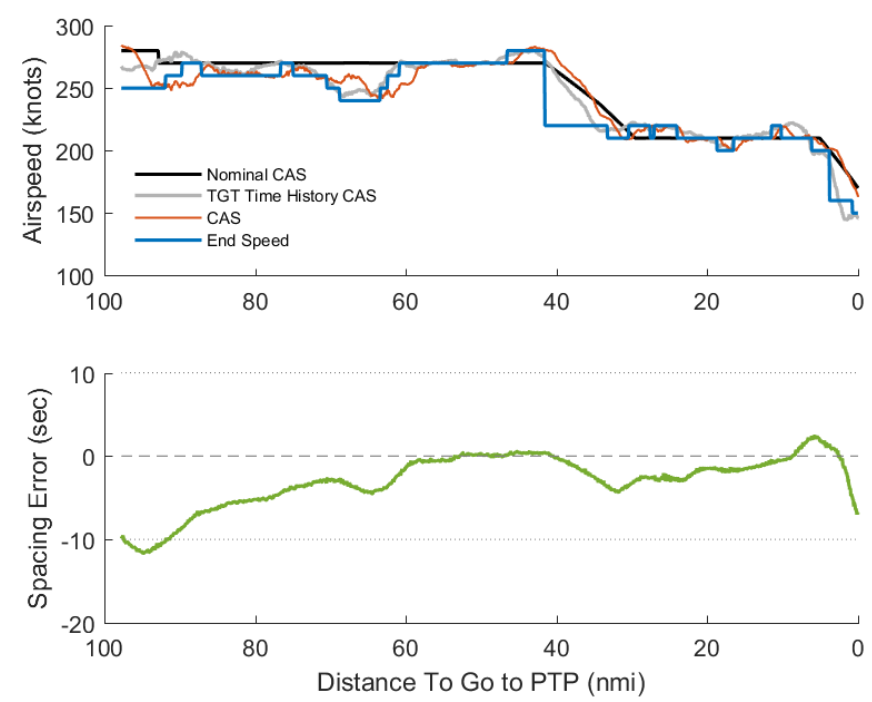

Fig. 12. High frequency of IM commanded speeds. 
In this particular case study, the IM speed changes from 78$60 \mathrm{nmi}$ were primarily caused by to the difference between the headwind component experienced by the Target and Ownship (Target airspeed constant but ground speed varied). Fig. 13 shows this difference in the headwind component was due to the difference in altitude of the two aircraft when flying the approach. The IM speed changes from 35-10 nmi were due to small perturbations from a range of factors, including variations in altitude again creating a difference in the headwind component experienced by each aircraft, and the variations in airspeed due to the tolerance of each aircraft's auto-throttle system.
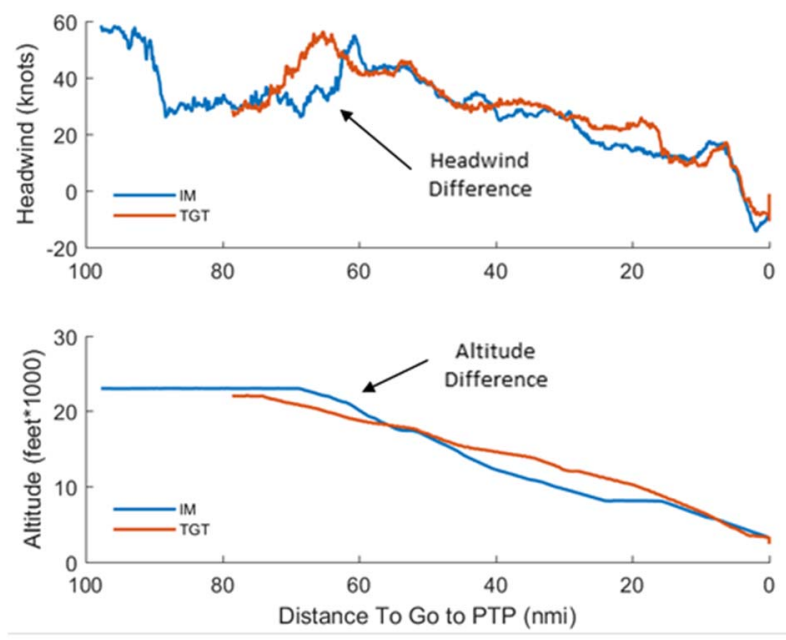

Fig. 13. Headwind component difference due to altitude variation.

To reduce the number of extraneous IM commanded speed changes caused by normal variations in aircraft speed, altitude, and headwind, the spacing algorithm needs a more sophisticated method to trade-off spacing error with the number of speed commands issued. In particular, the current proportional speed control law does not take advantage of the recorded Target aircraft time-history information between the Target's position and the Ownship's position. Options to explore include modifying the existing speed control logic by improving the filtering of the Target aircraft's time history speed, but potentially the most promising option would be to explore using more advanced control law techniques that could take advantage of the recorded Target aircraft time history data.

\section{Case Study 3: Expected versus Actual Decelration Rates}

The final case study examines the difference between the deceleration rate expected by the IM prototype and the aircraft's actual deceleration rate. The expected deceleration values used by the IM avionics prototype for both the Target and Ownship was 0.75 knots per second for level segments of the approach, and 0.50 knots per second for descending segments of the approach. While this approach complies with the requirements [15], it is a lower fidelity approximation of deceleration rates used by the NASA version of the spacing algorithm, which estimated deceleration rates based on the aircraft's speed, altitude, descent rate, and configuration.

Similar to the previous case study, the final $10 \mathrm{nmi}$ of Cross operation (Achieve phase using the TBO control law) in Fig. 14 shows the expected deceleration rate (light blue line), the IM aircraft's airspeed (red line), and the spacing error (green line). The aircraft decelerated faster than the IM prototype expected, therefore the Ownship moved aft relative to its current position, which drove the spacing error in the positive direction. Also similar to the previous case study, the TBO control law was prohibited from commanding a speed above the final nominal speed (170 knots) during the deceleration to ensure that the Ownship was able to achieve a stabilized approach.
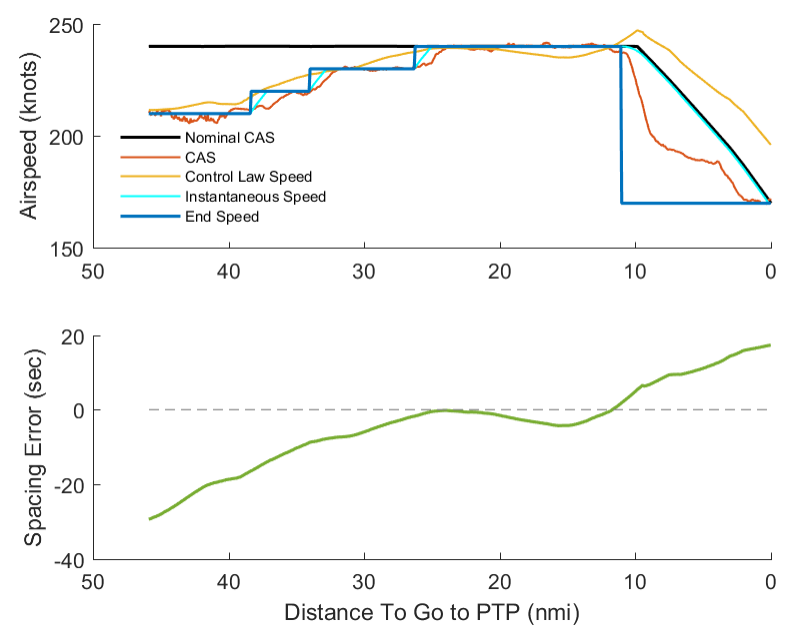

Fig. 14. Impact of expected versus actual deceleration rates.

The Ownship decelerating faster than the expected speed (red line below the yellow line) caused the spacing error to increase. The difference between the aircraft's deceleration rate and the expected deceleration rate was amplified by the size of the 70 knot speed change. Reducing the size of the speed change prior to the FAF would reduce the impact of different deceleration rates. Additionally, after the Target aircraft crosses the ABP, the spacing algorithm uses a nominal speed profile instead of the Target aircraft's state data. A more advanced predictive TBO speed control law could use this trajectory information more effectively to determine the IM desired speed.

\section{DISCUSSION}

The results presented in this paper indicate that improvements to the IM commanded speed behavior is needed before implementation into the National Airspace System. Pilot comments indicated that the acceptability would be improved if the rate of speed commands and rate of speed reversals were reduced. Statistical analysis of the IM commanded speeds supported the pilots' comments. Case studies were also presented to better understand the fundamental causes of the frequent speed changes and speed reversals.

The results suggest that the proportional speed control laws that are currently being used for IM operations may not be the best speed control law design for achieving ideal speed behavior while meeting the spacing objectives, particularly given the variability of normal flight operations. It is recommended that the IM research community investigate alternative speed control law designs that are more predictive in nature than the speed control laws used in the ATD-1 flight test prototype. More predictive speed control laws may be able to trade off speed command behavior and spacing error in a smarter way, minimizing the number of speed changes. For example, the CTD 
speed control law could use the recorded Target aircraft time history data to smooth out the speed behavior, and the TBO speed control law could use future decelerations on the published procedure to reduce the number of speed changes.

\section{RECOMMENDATIONS}

Alternative approaches and enhancements to the IM requirements are offered in this section of the paper. Some of these recommendations are already under investigation by the FAA and industry working groups.

\section{A. IM Spacing Requirements}

The first sub-section in Part V of this paper outlines how the IM spacing algorithm overwhelmingly met the desired performance requirements [15]; however, as flown in this flight test, the rate of speed changes and speed reversals during IM operations described in the remainder of Part V were too high. Recommendations to reduce the number of speed changes and speed reversals, as well as other improvements to the IM spacing algorithm, are summarized below.

- Expected acceleration and deceleration rates should more closely align to actual performance throughout the range of operations experienced by the IM equipped aircraft (altitude, airspeed, descent rate, as well as flap and gear configuration).

- Explore alternative control law techniques to allow for tradeoffs between spacing error and IM speed change behavior to reduce the rate of speed changes and speed reversals. In particular, better filtering of Target airspeed and the use of upcoming decelerations on the published procedures should be a priority.

- $\quad$ Reduce the spacing error correction rate when feasible to minimize the number of IM speed changes.

- Ensure indications presented to the pilots will result in a successful IM operation; provide indications to the pilots when the operation can no longer be achieved.

Speed control laws that are more advanced than those used in this flight test may be able to reduce the number of speed increases during arrivals by creating an optimal speed profile that resolves the spacing error while minimizing the number of speed changes, particularly speed increases [19]. Characterizing the speed change behavior of different speed control laws should be a focus of future IM research.

\section{B. IM Procedures and Operations}

Recommendations below are based on comments from the flight crews and research team observations. Recommendations to consider to simplify the IM procedures and make the IM operations more acceptable are summarized below.

- The arrival and approach procedures must support speed as the primary control method to achieve the schedule and desired spacing; specifically, as compared to the procedures used during this flight test, slightly shallower descents, slightly slower nominal airspeeds on final, and limiting the magnitude of speed changes to 40 knots on arrival and 20 knots on final.
- Explore alternatives to use the vertical path mode provided by the flight management computers; specifically, modify the aircraft behavior to remain in vertical path mode when IM airspeeds are set in the mode control panel, or if not feasible, set the IM airspeed into the flight management computer instead of the mode control panel when above 10,000 feet.

\section{REFERENCES}

[1] J. Brown, "NASA ATD-1 Avionics Phase 2 Flight Test Plan," NASA/CR2017-219595.

[2] D. Boyle, K. Rein-Weston, R. Berckefeldt, H. Eggling, C. Stankiewicz, and G. Silverman, "ATD-1 Avionics Phase 2 Flight Test: Flight Test Operations and Safety Report (FTOSR),"NASA/CR-2017-219592.

[3] K. Swieringa, S. Wilson, B. Baxley, R. Roper, T. Abbott, I. Levitt, and J. Scharl, "Flight Test Evaluation of the ATD-1 IM Application," $17^{\text {th }}$ AIAA Aviation Technology, Integration, and Operations Conference (ATIO), AIAA 2017-4094, Denver, CO, June 2017.

[4] B. Baxley, K. Swieringa, S. Wilson, R. Roper, C. Hubbs, P. Goess, and R. Shay, "Flight Crew Survey Responses from the Interval Management (IM) Avionics Phase 2 Flight Test," $17^{\text {th }}$ AIAA Aviation Technology, Integration, and Operations Conference (ATIO), AIAA 2017-4095, Denver, CO, June 2017.

[5] B. Baxley, H. Swenson, T. Prevot, and T. Callantine, "NASA's ATM Technology Demonstration-1: Integrated Concept of Arrival Operations," $31^{s t}$ Digital Avionics Systems Conference (DASC), IEEE/AIAA, Williamsburg, VA, 2012.

[6] B. Baxley, W, Johnson, J. Scardina, and R. Shay, "Air Traffic Management Technology Demonstration-1 Concept of Operations (ATD1 ConOps), Version 3.0," NASA/TM-2016-219213.

[7] B. Baxley, et. al., "Experiment Description and Results for Arrival Operations Using Interval Management with Spacing to Parallel Dependent Runways," NASA/TP-2013-217998.

[8] “Air Traffic Control,” JO 7110.65W, U.S. Department of Transportation, Washington, DC., Dec. 10, 2015.

[9] S. Wilson, J. Kibler, C. Hubbs, and J. Smail, "Air Traffic Management Technology Demonstration-1 Resarch and Procedural Testing of Routes," NASA/TM-2015-218707.

[10] B. Baxley, S. Wilson, K. Swieringa, W. Johnson, R. Roper, C. Hubbs, P. Goess, and R. Shay, "Human-in-the-Loop Assessment of Alternative Clearances in Interval Management Arrival Operations," NASA/TP2016-219362

[11] R. Roper, B. Baxley, K. Swieringa, and C. Hubbs, "Participant training for a flight test evaluation," $36^{\text {th }}$ Digital Avionics Systems Conference (DASC), IEEE/AIAA, St. Petersburg, FL, 2017.

[12] E. Alves, "FIM Avionics Operations Manual," NASA/CR-2017-219593.

[13] G. Wilber, "ATM Technology Demonstration-1 Phase II Boeing Configurable Graphical Display (CGD) Software Design Description," NASA/CR-2017-219594.

[14] T. Abbott, "An Overview of a Trajectory-Based Solution for En Route and Terminal Area Self-Spacing: Eighth Revision," NASA/CR-2017$\mathrm{xxxxxx}$, in press.

[15] RTCA SC-186, "Minimum Operational Performance Standards (MOPS) for Flight-deck Interval Management (FIM)," DO-361, Sept 2015.

[16] L. Weitz, W. Ganghuai, and H. Stassen, "Investigating the Performance of Two IM Algorithms in a Fast-time Simulation Environment," AIAA Guidance, Navigation, and Control Conference, Aug 2013.

[17] W. Penhallegon, R. Bone, and H. Stassen, "Results from a Field Evaluation of Interval Management during an Optimized Profile Descent Arrival and Approach," AIAA Guidance, Navigation, and Control Conference, San Diego, CA, Jan 2016.

[18] M. Hollander, and D. Wolfe, "Nonparametric Statistical Methods," John Wiley \& Sons, New York, NY $2^{\text {nd }}$ edition, 1999.

[19] T. Gaydos, and L. Weitz, "Designing Stochastic Optimal Control Laws for Interval Management," AIAA Guidance, Navigation, and Control Conference, San Diego, CA, Jan 201 